\title{
Control Method of a Rotary Blood Pump tor a Left Ventricular Assist Device
}

DOI: $10.17691 /$ stm2016,8.1.04

Received May 5, 2015

D.S. Petukhov, PhD Student, Department of Biomedical Systems;

D.V. Telyshev, PhD, Senior Researcher, Department of Biomedical Systems;

S.V. Selishchev, DSC, Professor, Head of the Department of Biomedical Systems

National Research University of Electronic Technology — MIET, 1 Shokin Square, Zelenograd, Moscow,

124498, Russian Federation

The aim of the investigation is to develop a control method of a rotary blood pump (RBP) to solve the following problems: estimation of the pump flow rate, achievement and maintaining of the desired flow level through the continuous adjustment of pump speed and prevention of adverse effects on the cardiovascular system.

Results. Functional chart of RBP control consists of several units: a unit for evaluation of instantaneous pump flow rate, unit for estimation of approximate and actual pump flow rate and identification of pumping states, and unit for speed adjustment forming a new speed value of the desired flow rate and current pumping state. The core of the functional chart is RBP unit presented by a mathematical model of RBP.

Waveforms of pump flow and speed changes and indices for RBP state identification are given in the work. Hemodynamic curves (the flow rate through aortic valve and minimum volume of the left ventricle during a cardiac cycle) are used to evaluate accuracy of pumping states identification. The possibility to adjust pump flow in various physiological conditions (variation of the heart rate and left ventricular contractility) is demonstrated. Control of the pumping states allows to avoid adverse conditions in the cardiovascular system, and to estimate physiological changes in its work such as aortic valve closure.

Conclusion. The proposed control method of a RBP allows to achieve and maintain the desired pump flow rate under various physiological conditions. This method is supposed to be used in the development of control system for the left ventricular assist devices.

Key words: ventricular assist device; rotary blood pump; adaptive rotary blood pump control.

One of the main requirements to the circulatory support system is supplying of the adequate cardiac output. Generally, this requirement is realized with the help of control algorithms or methods for the implanted part of circulatory support system, i.e. rotary blood pump (RBP).

Method of control using pressure difference across the pump for calculation of the pulsatility index is suggested in the work [1]. Depending on the aims of control, a certain value of the gradient of pulsatility index with respect to pump speed is assigned, providing either a highest feasible flow or an average flow with a controlled opening of the aortic valve (AV) without any negative effect on the cardiovascular system. Wang et al. [2] have developed a control algorithm for preventing ventricular collapse by maintaining differential pump speed above user-defined threshold value and providing a sufficient flow by maintaining a reference pressure difference between the left ventricle (LV) and aorta. In the work [3] a method of establishing a balance between a cardiac output of the right ventricle and a combined flow rate of the LV and a pump is suggested. The value of pump flow pulsatility is used as feedback parameter in order to adjust the RBP flow.
The aim of the investigation is to develop a control method of a rotary blood pump to solve the following problems: estimation of the pump flow rate, achievement and maintaining of the desired flow rate through the continuous adjustment of the pump speed and prevention of adverse effects on the cardiovascular system.

Methods. Pump flow rate estimation is performed using mathematical model of RBP, which takes into account inertial and viscous properties of blood. Adverse effect on the cardiovascular system is prevented by controlling pumping states (backflow of blood through the pump $P_{B F}$, partial assist of the ventricle with a periodically opening aortic valve $P_{P A}$, full assist of the ventricle with a constantly closed aortic valve $P_{F A}$, partial and full ventricular collapse during cardiac cycle $P_{P V C}$ and $P_{F V C}$ ).

A functional chart of the proposed control method is presented on Figure 1. The main part of the functional chart is a RBP unit. The principal component of this unit is a mathematical model of the RBP, described by the following equation:

$$
L \frac{d Q}{d t}=a Q+b Q^{2}+c Q^{3}+d \omega^{2}+e Q \omega^{2}+f Q^{2} \omega+g-H,
$$


where $L$ is the parameter, characterizing blood inertia in the given pump, which equals to $0.2 \mathrm{~mm} \mathrm{Hg} \cdot \mathrm{min}^{2} \cdot \mathrm{L}^{-1} ; Q$ is pump flow rate $(\mathrm{L} / \mathrm{min}) ; \omega$ is pump speed $\left(\mathrm{min}^{-1}\right) ; H$ is pressure difference across the pump $(\mathrm{mm} \mathrm{Hg}) ; a-g$ are coefficients, obtained by optimization on the basis of Levenberg-Marquardt method (their values are given in Table 1), each coefficient being related to the blood viscosity $\mu(\mathrm{cP})$ by the following linear relationship: $y(\mu)=k \cdot \mu+x$.

Thus, the pump flow rate at a given time $Q(t)$ is calculated on the basis of speed value $\omega$, pressure difference $H$ and blood viscosity $\mu$, which value is set on the external control console.

The estimation unit of the functional chart is designed for storing the calculated value of $Q(t)$, evaluation of the approximate and actual flow rate and identification of RBP states. Approximate flow $Q_{A}$ is counted as a blood volume, pumped over by the pump during the time equal to nine cardiac cycles $(6.75 \mathrm{~s}$ at a heart rate $(\mathrm{HR})$ 80 beats per minute $(\mathrm{bpm})$ ); the obtained value is converted in liters per minute. The number of cardiac cycles necessary for estimation of the approximate flow may be arbitrary; in our case it was chosen to be nine in order to approximately evaluate a minute pump flow and to adjust it quickly if physiological conditions change. The actual flow $Q_{P}$ is a blood volume pumped over by the pump per minute.

\begin{tabular}{|c|}
\hline $\begin{array}{l}\text { Coefficients of rotary blood } \\
\text { pump model }\end{array}$ \\
\hline $\begin{array}{c}a=a_{1}+a_{2} \cdot \mu \\
a_{1}=-6.2332 \mathrm{~mm} \mathrm{Hg} \cdot \mathrm{L}^{-1} \\
a_{2}=-0.0254 \mathrm{~mm} \mathrm{Hg} \cdot \mathrm{L}^{-1} \cdot \mathrm{cP}-1\end{array}$ \\
\hline $\begin{array}{c}b=b_{1}+b_{2} \cdot \mu \\
b_{1}=0.5339 \mathrm{~mm} \mathrm{Hg} \cdot \mathrm{L}^{-2} \\
b_{2}=-0.0239 \mathrm{~mm} \mathrm{Hg} \cdot \mathrm{L}^{-2} \cdot \mathrm{cP}-1\end{array}$ \\
\hline $\begin{array}{c}C=C_{1}+C_{2} \cdot \mu \\
C_{1}=-0.1594 \mathrm{~mm} \mathrm{Hg} \cdot \mathrm{L}^{-3} \\
C_{2}=-0.0147 \mathrm{~mm} \mathrm{Hg} \cdot \mathrm{L}^{-3} \cdot \mathrm{CP}-1\end{array}$ \\
\hline $\begin{array}{c}d=d_{1}+d_{2} \cdot \mu \\
d_{1}=1.0778 \mathrm{~mm} \mathrm{Hg} \cdot \mathrm{min}^{2} \\
d_{2}=0.0495 \mathrm{~mm} \mathrm{Hg} \cdot \mathrm{min}^{2} \cdot \mathrm{cP}^{-1}\end{array}$ \\
\hline $\begin{array}{c}e=e_{1}+e_{2} \cdot \mu \\
e_{1}=-0.0788 \mathrm{~mm} \mathrm{Hg} \cdot \mathrm{min}^{2} \cdot \mathrm{L}^{-1} \\
e_{2}=-0.0133 \mathrm{~mm} \mathrm{Hg} \cdot \mathrm{min}^{2} \cdot \mathrm{L}^{-1} \cdot \mathrm{cP}-1\end{array}$ \\
\hline $\begin{array}{c}f=f_{1}+f_{2} \cdot \mu \\
f_{1}=0.1568 \mathrm{~mm} \mathrm{Hg} \cdot \mathrm{min} \cdot \mathrm{L}^{-2} \\
f_{2}=0.0263 \mathrm{~mm} \mathrm{Hg} \cdot \mathrm{min} \cdot \mathrm{L}^{-2} \cdot \mathrm{CP} \mathrm{P}^{-1}\end{array}$ \\
\hline $\begin{array}{c}g=g_{1}+g_{2} \cdot \mu \\
g_{1}=-0.6583 \mathrm{~mm} \mathrm{Hg} \\
g_{2}=-0.6671 \mathrm{~mm} \mathrm{Hg} \cdot \mathrm{CP}^{-1}\end{array}$ \\
\hline
\end{tabular}

Table 2

Indices for identification of rotary blood pump states

$\begin{array}{ll}S_{B F} & -2 \cdot \min \frac{d^{2} Q}{d t^{2}} \cdot \frac{d Q}{d \mu} /\left(\max \frac{d^{2} Q}{d t^{2}} \cdot \frac{d Q}{d \mu}-\min \frac{d^{2} Q}{d t^{2}} \cdot \frac{d Q}{d \mu}\right) \\ S_{A V} & -2 \cdot \min \frac{d Q}{d t} \cdot \frac{d Q}{d \mu} /\left(\max \frac{d Q}{d t} \cdot \frac{d Q}{d \mu}-\min \frac{d Q}{d t} \cdot \frac{d Q}{d \mu}\right) \\ S_{P V C} & \max \frac{d Q}{d t} \cdot \frac{d Q}{d \omega} \\ S_{F V C} & -2 \cdot \min \frac{d Q}{d t} /\left(\max \frac{d Q}{d t}-\min \frac{d Q}{d t}\right)\end{array}$

Pumping states are identified by the analysis of changes in the dynamics of derivatives, obtained from the pump mathematical model. To simplify the description of these changes some indices were introduced: e.g. $S_{B F}$ uses to identify backflow of blood through the pump, $S_{A V}$ for identification of partial and full assist of LV, $S_{P V C}$ and $S_{F V C}$ for partial and full collapse of the ventricle during cardiac cycle. A list of used indices and their values is given in Table 2. A detailed description of the RBP mathematical model and method of pumping states identification is presented in work [4].

A new speed value $\omega(t+1)$ is formed in the speed adjustment unit. It depends on the difference between the approximate and desired flow $Q_{D}$ and on the current pumping state. If $Q_{A}$ and $Q_{D}$ do not correspond, the pump speed will change with an increment of $100 \mathrm{rpm}$ until their matching is set. When some undesired pumping state is identified $\left(P_{B F}\right.$ or $\left.P_{F V C}\right)$, which adverse effects on the cardiovascular system, the speed is forcibly increased or decreased independently of the pump flow at this moment.

The development and testing of the RBP control method was carried out using the mathematical model of the cardiovascular system in which RBP was connected between LV and aorta [4]. All results were obtained at blood viscosity value $\mu=3.6 \mathrm{cP}$.

Results and Discussion. A waveform of flow rate 
$Q_{P}$ and $Q_{A}(\mathrm{~L} / \mathrm{min})$, pump speed $\omega_{p}(\mathrm{rpm} / 1,000)$ and indices for identification of pumping states at desired flow rate $Q_{D}=4.5 \mathrm{~L} / \mathrm{min}$ is presented in Figure 2. After estimation of the approximate flow $Q_{A}$ it is compared with the desired $Q_{D}$ and, if necessary, the RBP speed is changed; in this case the speed increases by $100 \mathrm{rpm}$ every time.

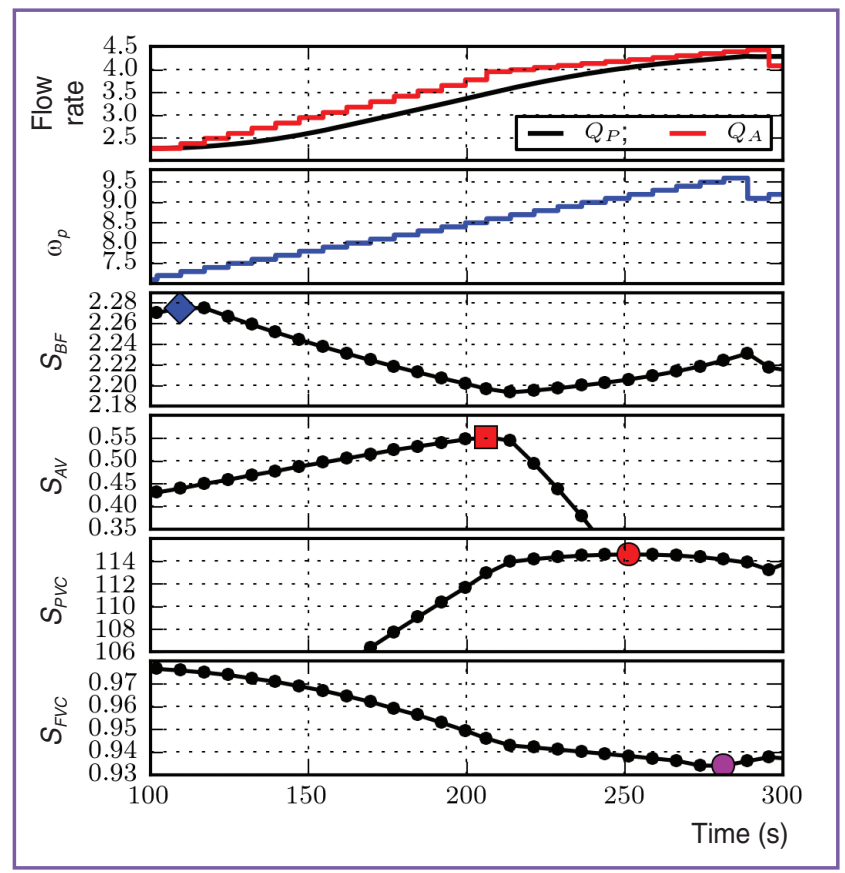

Figure 2. A waveform of pump flow $Q_{P}$ and $Q_{A}(L / m i n)$, pump speed $\omega_{p}(\mathrm{rpm} / 1,000)$ and indices $S_{B F}, S_{A V}, S_{P V C}$ and $S_{F V C}$ for $Q_{D}=4.5 \mathrm{~L} / \mathrm{min}$
As seen on the figure, the increase of RBP speed results in a certain dynamics of every index. Thus, partial assist of the ventricle $P_{P A}$ corresponds to the decrease of $S_{B F}$ index and increase of $S_{A V}$, while partial ventricular collapse during a cardiac cycle corresponds to the decrease of $S_{P V C}$ and $S_{F V C}$ indices. Transitions from one state to another, which characterized by the changes in index dynamics, are pointed by color markers: a blue diamond-shaped marker on $S_{B F}(t)$ designates the moment of transition from $P_{B F}$ state to the state of partial LV unloading $P_{P A}$. A red square marker on $S_{A V}(t)$ marks the moment of constant AV closure, which corresponds to the transition from partial assist state to the full assist state $P_{F A}$.

A red round marker on $S_{P V C}(t)$ corresponds to the transition in $P_{P V C}$ state, denoting a partial collapse of the ventricle during systole. A violet round marker on $S_{F V C}(t)$ corresponds to the transition in $P_{F V C}$ state, in this case the pump speed reduces by $500 \mathrm{rpm}$. As the desired flow level was not achieved, pump speed continues to increase.

A waveform of pump flow $Q_{P}$ and $Q_{A}$ (L/min), pump speed $\omega_{p}(\mathrm{rpm} / 1,000)$, flow through the aortic valve $Q_{A V}$ (L/min), $S_{A V}$ index for the selected value $Q_{D}=3.8 \mathrm{~L} / \mathrm{min}$ at LV contractility changes $C_{L V}(\%)$ is shown on Figure 3. In this case the reduction of $C_{L V}$ by $10 \%$ does not alter the pump speed, which does not allow to detecting AV closure and transition to the $P_{F A}$ state. To trace the influence of such physiological changes, a differential index $\Delta S_{A V}$ was introduced, which is described by the following equation:

$$
\Delta S_{A V}=\left(S_{A V}[i]-S_{A V}[i-1]\right)-\left(S_{A V}[i-1]-S_{A V}[i-2]\right),
$$

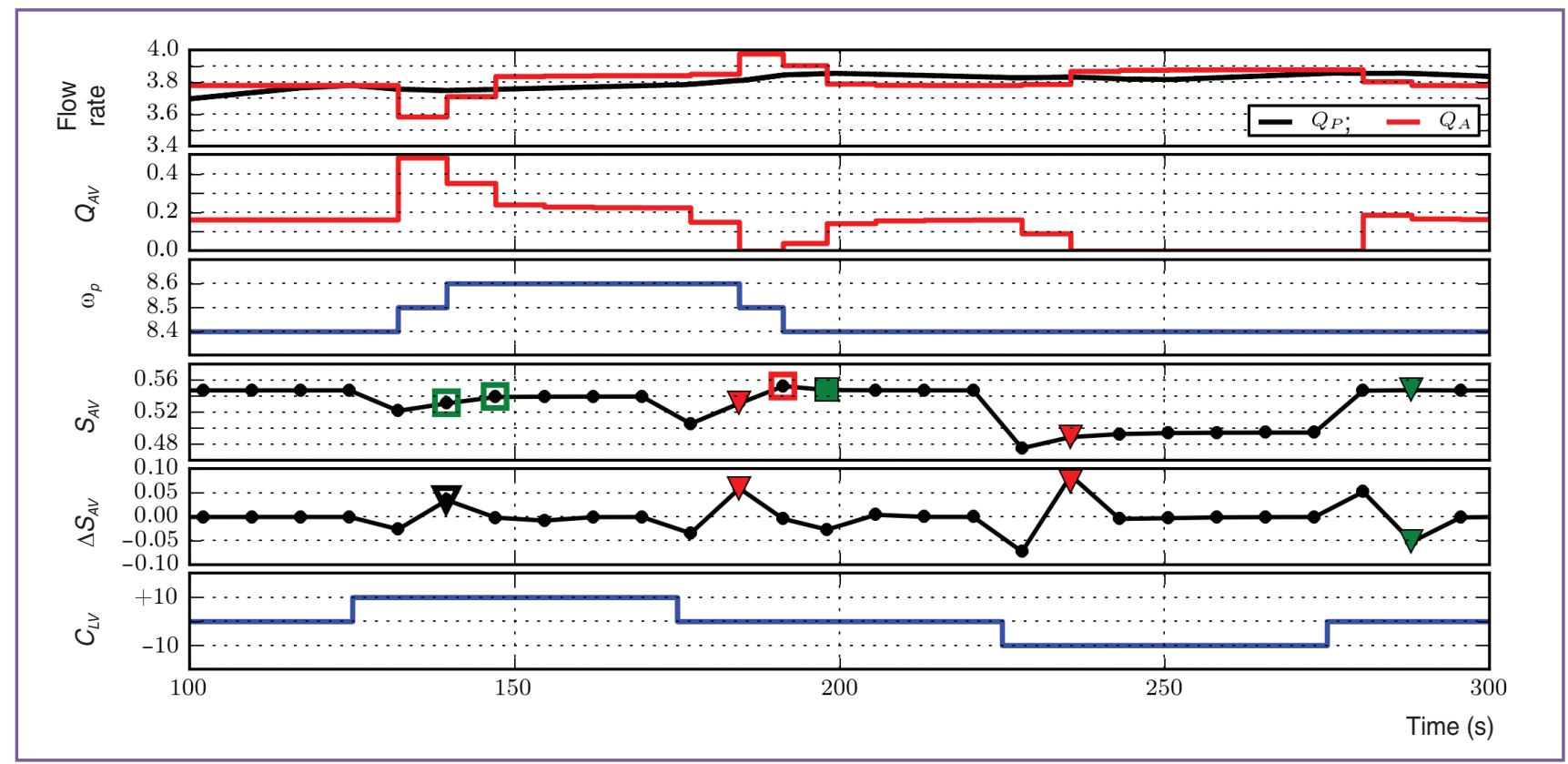

Figure 3. A waveform of pump flow $Q_{P}$ and $Q_{A}(\mathrm{~L} / \mathrm{min})$, pump speed $\omega_{p}$ (rpm/1,000), flow through the aortic valve $Q_{A V}(\mathrm{~L} / \mathrm{min}), S_{A V}$ and $\Delta S_{A V}$ indices for $Q_{D}=3.8 \mathrm{~L} / \mathrm{min}$ at left ventricle contractility changes $C_{L V}(\%)$ 
where $i$ is the time step corresponded to estimation of current approximate flow $Q_{A} ; i-1$ is the estimation of previous $Q_{A}$ value.

The increase of contractility by $10 \%$ is resulted in increase of pump speed due to the augmentation of AV flow, which is shown on $Q_{A V}(t)$. The increase of $S_{A V}$ index with the consecutive increase of speed by $200 \mathrm{rpm}$ corresponds to the partial assist pumping state $P_{P A}$ and AV opening, which is indicated by empty green square markers. At the same time, change of $\Delta S_{A V}$ is not considered - it is pointed by an empty black triangle marker on $\Delta S_{A V}(t)$.

The next characteristic change of $\Delta S_{A V}$ is related to the reduction of LV contractility to the initial level. Such alteration simultaneously with the decrease of $S_{A V}$ index corresponds to the AV closure and transition to $P_{F A}$ state; it is pointed by a red triangular marker. Following decrease of speed by $100 \mathrm{rpm}$ corresponds to the state of full assist state also due to the increase of $S_{A V}$ index at the decrease of the pump speed. However following reduction of the speed by $100 \mathrm{rpm}$ and decrease of $S_{A V}$ index denotes the change in index dynamics and corresponds to the transition from the $P_{F A}$ state to the state of partial LV unloading (pointed by a green square marker).

The decrease of $\mathrm{C}_{L V}$ by $10 \%$ does not lead to the speed change, therefore $\Delta S_{A V}$ index is used to trace the effect of this physiological change on the pumping state. In this case the characteristic change of $\Delta S_{A V}$ from the negative to positive value at a decreased $S_{A V}$ corresponds to the constant AV closure and to the transition in $P_{F A}$ state; it is pointed by a red triangular marker.
The increase of contractility to the initial value results in increase of $S_{A V}$ and the characteristic change of $\Delta S_{A V}$, which corresponds to the transition in partial assist state and pointed by a green triangular marker on $S_{A V}(t)$.

A waveform of pump flow $Q_{P}$ and $Q_{A}(L / \mathrm{min})$, pump speed $\omega_{p}$ (rpm/1000), flow through the aortic valve $Q_{A V}(\mathrm{~L} / \mathrm{min}), S_{A V}$ and $\Delta S_{A V}$ indices for $Q_{D}=3.8 \mathrm{~L} / \mathrm{min}$ $\mathrm{HR}$ changes demonstrates, that decrease of $\mathrm{HR}$ to $70 \mathrm{bpm}$ does not alter the pump speed (Figure 4). In this case $\Delta S_{A V}$ index is also used to determine effect of physiological changes on the pumping state - its characteristic change in case of $S_{A V}$ decrease allows to detect AV closure (pointed by a red triangular marker).

Increase of $S_{A V}$ at a characteristic change of $\Delta S_{A V}$, which is the opposite of the previous one, corresponds to the transition in $P_{P A}$ state and AV opening (marked by a green triangle). With further HR changes pump speed at first increases and then reduces by $100 \mathrm{rpm}$. In the first case the speed increase is accompanied by the increase of $S_{A V}$ index, in the second one speed decrease results in the decrease of the index. Both of these changes corresponds to the $P_{P A}$ state and, therefore, are pointed by the empty square green markers.

A waveform of pump flow $Q_{P}$ and $Q_{A}$ (L/min), pump speed $\omega_{p}(\mathrm{rpm} / 1,000)$, minimum volume of LV during a cardiac cycle $V_{L V \text { min] }}(\mathrm{ml})$ and $S_{F V C}$ and $\Delta S_{F V C}$ indices for $Q_{D}=4.4 \mathrm{~L} / \mathrm{min}$ at $\mathrm{HR}$ changes illustrates, that decrease of HR to $70 \mathrm{bpm}$ leads to the increase of $S_{F V C}$ index (Figure 5). In spite of the fact that increase of $S_{F V C}$ with the increase of pump speed corresponds to the transition in $P_{F V C}$ state, this change is not associated with the transition to $P_{F V C}$ due to the characteristic change of

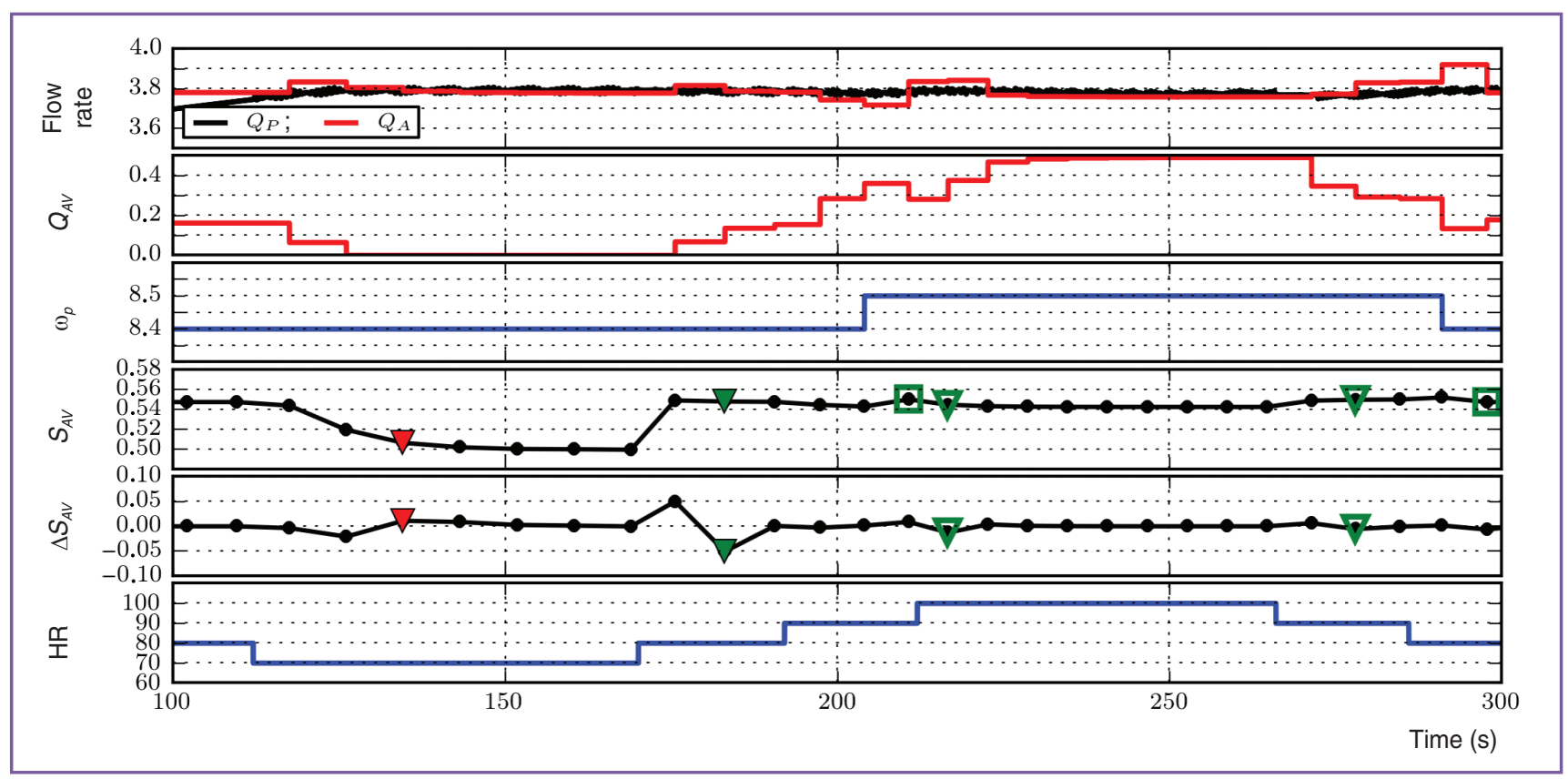

Figure 4. A waveform of pump flow $Q_{P}$ and $Q_{A}$ (L/min), pump speed $\omega_{p}(\mathrm{rpm} / 1,000)$, flow through the aortic valve $Q_{A V}(\mathrm{~L} / \mathrm{min}), S_{A V}$ and $\Delta S_{A V}$ indices for $Q_{D}=3.8 \mathrm{~L} / \mathrm{min}$ at heart rate $(\mathrm{HR})$ changes $(\mathrm{bpm})$ 


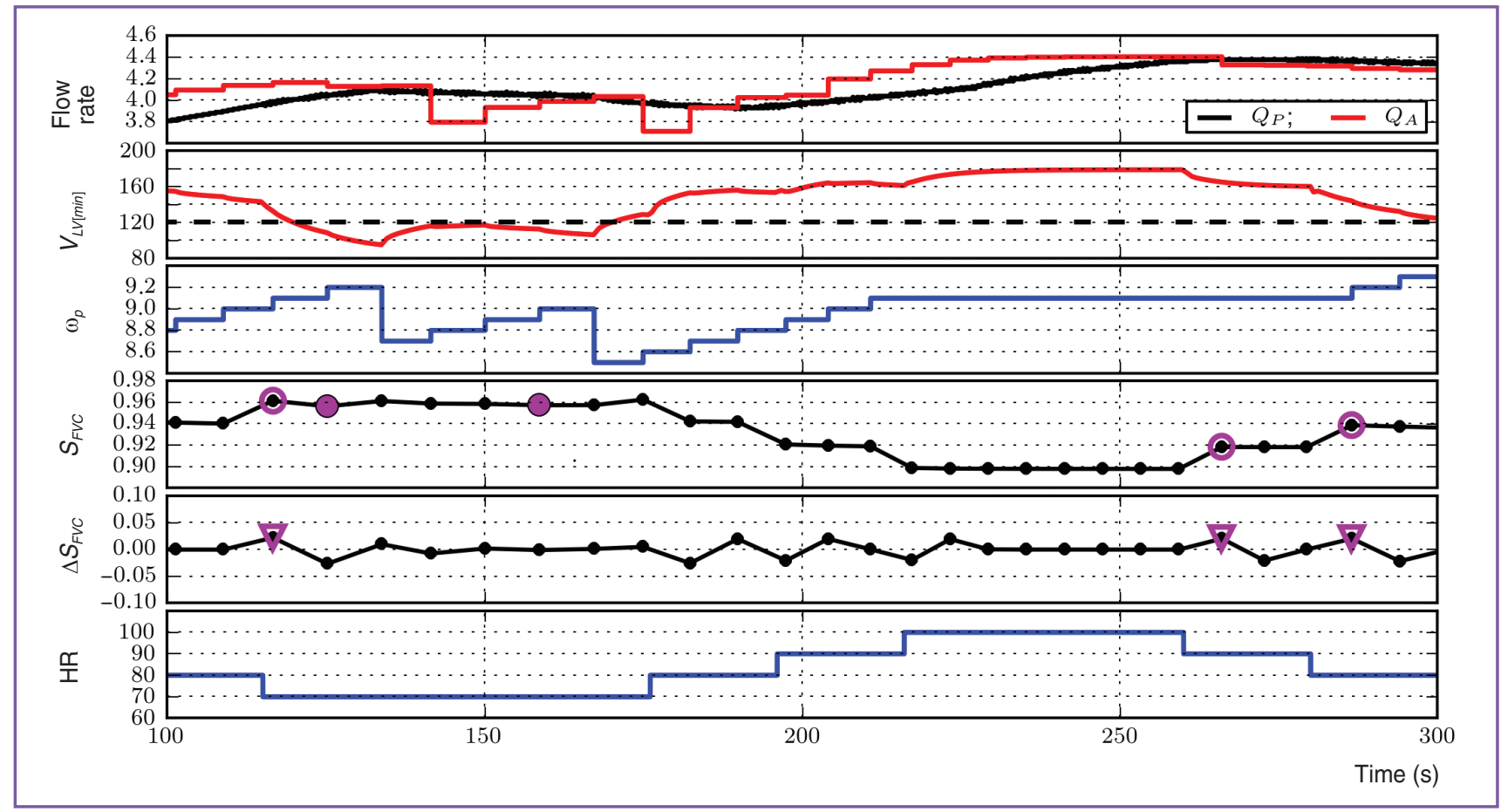

Figure 5. A waveform of pump flow $Q_{P}$ and $Q_{A}(\mathrm{~L} / \mathrm{min})$, pump speed $\omega_{p}$ (rpm/1,000), minimum volume of left ventricle during a cardiac cycle $V_{L V[\min ]}(\mathrm{ml})$ and $S_{F V C}$ and $\Delta S_{F V C}$ indices for $Q_{D}=4.4 \mathrm{~L} / \mathrm{min}$ at heart rate $(\mathrm{HR})$ changes (bpm)

$\Delta S_{F V C}$. The situation like this is pointed by empty violet markers over the whole time range (by the round markers on $S_{F V C}(t)$, and triangular markers on $\left.\Delta S_{F V C}(t)\right)$.

The increase of $S_{F V C}$ with the increase of speed in other cases corresponds to the transition in full ventricular collapse $\left(P_{F V C}\right)$ state, i.e. to the decrease of volume lower reference value $(120 \mathrm{ml})$, that corresponds to the zero pressure in the ventricle - in this situation pressure in the ventricular chamber during systolic phase is constantly negative. The moment of transition to this state is pointed by round violet markers on $\Delta S_{F v c}(t)$, the pump speed also decreasing by $500 \mathrm{rpm}$.

It should be noted, that increase of HR to $100 \mathrm{bpm}$ allows reaching the desired flow level without any LV collapse, which is seen at $V_{L V[\min ]}(t)$.

Thus, the proposed control method of a RBP allows to achieve the desired flow rate under various physiological conditions by continuous adjustment of pump speed. The estimation of the RBP performance is carried out by means of the pump mathematical model, which uses values of blood viscosity, pressure difference across the pump and pump speed. The control of RBP states allows to avoid adverse states in the cardiovascular system associated with backflow of blood through the pump or full LV collapse. A long-term RBP operation in the full assist state $\left(P_{F A}\right)$ results in the valve leaflet fusion and thrombus formation, therefore assessment of the AV condition is also necessary [5].

The proposed control method has been tested under changing LV contractility and HR. The results demonstrate feasibility of achievement and maintaining of RBP flow rate on the required level and control adverse states in the cardiovascular system under varying physiological conditions. Identification of RBP states is possible in all considered cases, including a case of constant pump speed.

Conclusion. The presented control method of a rotary blood pump provides the desired flow rate and prevents adverse effects on the cardiovascular system under different physiological conditions. This method is supposed to be used in the development of adaptive control system for the left ventricular assist devices.

Study Funding. The study was supported by the grant of the Russian Scientific Fund (project No.14-3900044).

Conflicts of Interest. The authors declare no conflicts of interest related to this study.

\section{References}

1. Arndt A., Nüsser P., Graichen K., Müller J., Lampe B. Physiological control of a rotary blood pump with selectable therapeutic options: control of pulsatility gradient. Artif Organs 2008; 32(10): 761-771, http://dx.doi.org/10.1111/j.1525-1594. 2008.00628.x.

2. Wang Y., Koenig S.C., Slaughter M.S., Giridharan G.A. Rotary blood pump control strategy for preventing left ventricular suction. ASAIO J 2015; 61(1): 21-30, http://dx.doi.org/10.1097/ MAT.0000000000000152. 
3. Bakouri M.A., Salamonsen R.F., Savkin A.V., AlOmari A.H., Lim E., Lovell N.H. A sliding mode-based starling-like controller for implantable rotary blood pumps. Artif Organs 2014; 38(7): 587-593, http://dx.doi.org/10.1111/aor. 12223.

4. Petukhov D.S., Telyshev D.V. Simulation of changes in the dynamics of blood flow through the implantable axial flow pump. Meditsinskaya tekhnika 2014; 6: 44-47.

5. Granegger M., Schima H., Zimpfer D., Moscato F. Assessment of aortic valve opening during rotary blood pump support using pump signals. Artif Organs 2014; 38(4): 290-297, http://dx.doi.org/10.1111/aor.12167. 